\title{
Impacto de la pandemia por SARS-CoV-2 en la administración de vacunas del Calendario Nacional de Inmunizaciones en menores de 2 años
}

\author{
Impact of the SARS-CoV-2 pandemic on the administration of vaccines as per \\ the national immunization schedule in children younger than 2 years
}

\author{
Dr. Fernando Torres ${ }^{a}$, Dra. Paula Dominguez ${ }^{a}$ Dra. M. Eugenia Aruanno ${ }^{a}$ Dra. M. Julia Macheretta, \\ Dra. Eliana S. Nocent ${ }^{a}$, Dra. Lucía Risoli ${ }^{a}$ Dra. Magdalena Sasso ${ }^{a}$, Dra. Carmen Cabello y Dra. María N. Seoane ${ }^{b}$
}

\section{RESUMEN}

Introducción. El menor número de consultas a los centros de atención desde el comienzo de la pandemia por SARS-CoV-2 podría afectar la vacunación obligatoria.

Objetivo. Evaluar el impacto de la pandemia por SARS-CoV-2 en la administración de vacunas pentavalente y triple viral a niños menores de 2 años en el vacunatorio de un hospital pediátrico de la Ciudad de Buenos Aires.

Método. Estudiotransversal, queutilizóregistrosinformatizados del vacunatorio, de enero a mayo de 2019 y 2020.

Resultados. Desde la segunda quincena de marzo de 2020, se observó un $64,2 \%$ de disminución en la aplicación de vacunas. $\mathrm{Al}$ examinar la primera dosis de pentavalente y triple viral, la reducción fue del 74,9 \% y del 55,1\%, respectivamente.

Conclusión. A partir de la segunda quincena de marzo de 2020, se observó una disminución del $64,2 \%$ en las vacunas aplicadas respecto del mismo período en el año anterior.

Palabras clave: vacunas, pandemias, infecciones por coronavirus, prevención primaria, epidemiología.

http: / / dx.doi.org/10.5546/ aap.2021.198

Texto completo en inglés:

http:/ / dx.doi.org/10.5546/ aap.2021.eng.198

Cómo citar: Torres F, Domínguez P, Aruanno ME, Macherett MJ, et al. Impacto de la pandemia por SARS-CoV-2 en la administración de vacunas del Calendario Nacional de Inmunizaciones en menores de 2 años. Arch Argent Pediatr 2021;119(3):198-201.

a. Comité de Docencia e Investigación.

b. División Promoción y Protección de la Salud.

Hospital General de Niños Pedro de Elizalde (HGNPE),

Ciudad Autónoma de Buenos Aires, Argentina.

Correspondencia:

Dr. Fernando Torres: fernandoadriantorres@gmail.com

Financiamiento: Ninguno.

Conflicto de intereses: Ninguno que declarar.

Recibido: 21-7-2020

Aceptado: 27-10-2020

\section{INTRODUCCIÓN}

En respuesta a la pandemia por coronavirus de tipo 2 causante del síndrome respiratorio agudo grave (severe acute respiratory syndrome coronavirus 2; SARS-CoV-2, por sus siglas en inglés), la Argentina instauró el "aislamiento social preventivo y obligatorio" desde el 20 de marzo de 2020. ${ }^{1}$ Sus características impactaron en la sociedad de diversas maneras a lo largo del mundo. La asistencia a los centros de salud se vio afectada, con disminución de las consultas en el Departamento de Urgencias. ${ }^{2}$ Incluso una serie reportada por Snapiri y col. muestra el retraso en el diagnóstico de apendicitis. ${ }^{3}$ Es probable que lo mismo haya ocurrido con otros aspectos de la salud infantil.

Recientemente, se ha comunicado que la vacunación de los niños ha sido afectada por la pandemia. McDonald, en Inglaterra, encontró un descenso en la aplicación de vacunas hexavalente y triple viral (measles, mumps, and rubella; $M M R$, por sus siglas en inglés) del 6,7\% y del 19,8\%, respectivamente, al comparar el período de aislamiento preventivo con el mismo período de 2019. ${ }^{4}$ En Estados Unidos, Santoli y col., encontraron resultados similares al examinar dos fuentes del Centers for Disease Control and Prevention. ${ }^{5}$

Aunque es razonable pensar que, en la Argentina, pudo haber ocurrido un fenómeno similar, hasta el momento, no se encontró información al respecto. Contar con datos sobre la falta de adherencia al plan nacional de inmunizaciones es de vital importancia para la toma de conductas al respecto, pues limita el riesgo de brotes de enfermedades que pueden ser prevenidas por vacunas.

\section{OBJETIVO}

Evaluar el impacto de la pandemia por SARS-CoV-2 en la administración de la vacuna pentavalente y triple viral a niños menores de 
2 años en el vacunatorio de un hospital pediátrico de la Ciudad de Buenos Aires.

\section{MÉTODO}

Estudio transversal, que utilizó los registros informatizados del vacunatorio del Hospital General de Niños Pedro de Elizalde. La muestra se conformó con los registros de las vacunas administradas correspondientes al Calendario Nacional de Vacunación, en menores de dos años, en dos períodos similares: de enero a mayo de 2019 y de 2020.

Se registró el total de vacunas aplicadas, la primera dosis de la vacuna pentavalente y la primera dosis de la vacuna triple viral (que no correspondiera a campaña), por quincena, en ambos períodos, y se calculó la diferencia entre ambos períodos, que se expresó en porcentaje. Se eligieron las vacunas, pentavalente y triple viral por ser consideradas indicadoras de acceso al sistema y, además, por existir antecedentes sobre su evaluación en la misma situación. ${ }^{4}$ También se registró si los vacunados residían o no en la jurisdicción del Hospital (Ciudad de Buenos Aires).

El análisis se efectuó con SPSS 20.0 para Windows. Se solicitó y obtuvo la autorización del Comité de Ética en Investigación del Hospital. Toda información se trató de manera disociada de cualquier dato filiatorio.

\section{RESULTADOS}

En el período enero-mayo, en los menores de 2 años de edad, se aplicaron 7263 vacunas en
2019 y 5407 en 2020. Al comparar ambos años por quincena, se observó que el total de vacunas aplicadas fue similar hasta la segunda quincena del mes de marzo (aislamiento en la Argentina a partir del 20/3/2020), momento a partir del cual se observó una disminución del 64,2\% (intervalo de confianza-IC- del $95 \%$ : 63,2-66,1) (Figura 1).

Algo similar ocurrió con la administración de la primera dosis de pentavalente (difteria-tétanostos ferina -DPT-, Haemophilus influenzae tipo bHib-, hepatitis B -HB-) y de vacuna $M M R$, en las que la reducción fue del 74,9\% (IC 95\%: 72,3-76,5) y del 55,1\% (IC $95 \%$ : 48,2-61,4), respectivamente (Figuras 2 y 3 ).

Al evaluar el lugar de residencia de la población vacunada, se observó que, desde la segunda quincena de marzo, la cantidad de sujetos no residentes en la Ciudad de Buenos Aires vacunados disminuyó el 81,1\% (IC $95 \%$ : $79,7-82,5$ ) entre 2019 y 2020, mientras que, para los residentes, fue solo del 24,7 \% (IC $95 \%$ : 22,4-27,1).

\section{DISCUSIÓN}

En nuestro estudio, se observó una disminución en la aplicación de las vacunas del plan nacional de inmunizaciones a partir del momento del inicio del aislamiento social preventivo y obligatorio decretado por la pandemia por coronavirus 2019 (coronavirus disease 2019; COVID-19, por sus siglas en inglés). Estos datos coincidieron con los reportados por Santoli y col., quienes observaron una disminución de, al menos, el $50 \%$ en la aplicación de vacunas en las semanas siguientes a la

FIGURA 1. Variación quincenal del total de vacunas administradas 2019-2020

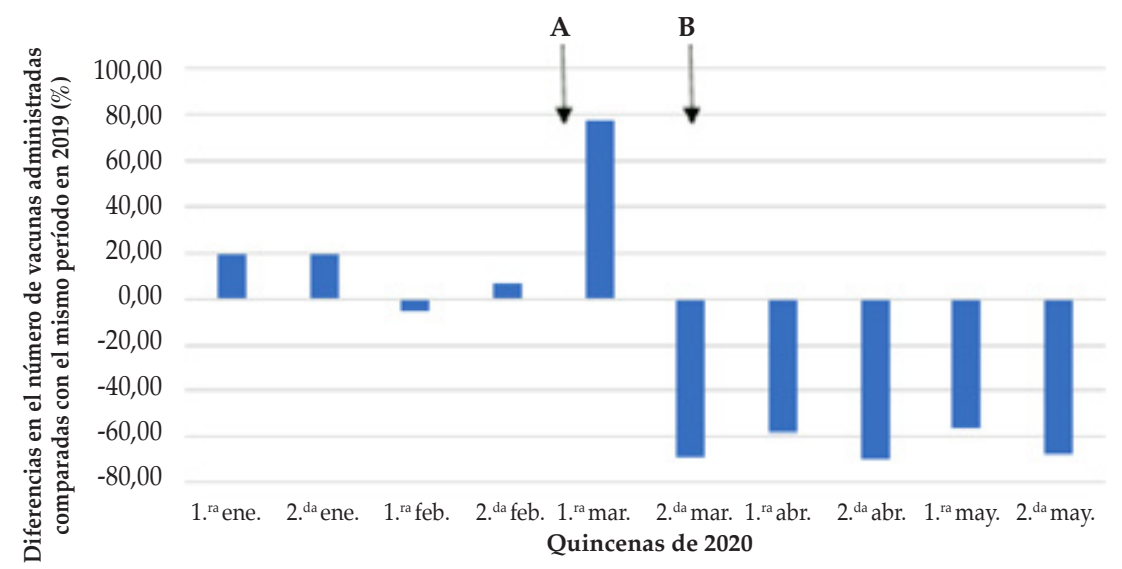

A. 3/3/20: primer caso de COVID-19.

B. 20-3-20: comienzo del aislamiento social. 
declaración de la emergencia nacional en Estados Unidos. ${ }^{5}$ Bramer y col., también encontraron que la cobertura en vacunación fue menor en el año 2020, al compararlo con el mismo período de 2016-2019, excepto para las vacunas del recién nacido que, habitualmente, se aplican en las maternidades. ${ }^{6}$

Al analizar el comportamiento de la administración de la primera dosis de vacuna pentavalente, en nuestra población, se notó un descenso más marcado y sostenido que el reportado por McDonald en Inglaterra. ${ }^{4}$ Esto podría estar relacionado con las características de la población que suele concurrir a nuestro Hospital, mayoritariamente, no residentes en la Ciudad de Buenos Aires, con la consecuente imposibilidad de utilizar el transporte público debido a las restricciones existentes. ${ }^{7}$ Esta presunción se ve reforzada por el hecho de que la asistencia al Departamento de Urgencias de

FIGURA 2. Variación quincenal de vacuna pentavalente administrada. Período 2019-2020

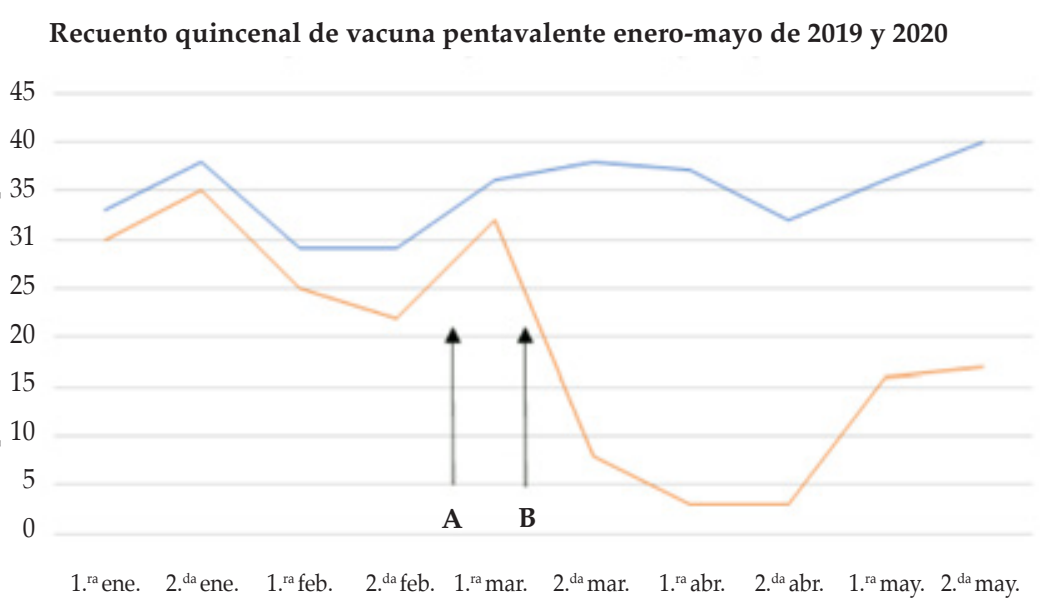

A. Comienzo de clases.

$-2019-2020$

B. Comienzo del aislamiento social preventivo.

FIGURA 3.Variación quincenal de vacuna triple viral MMR administrada. Período 2019-2020

Recuento quincenal de vacuna MMR enero-mayo de 2019 y 2020
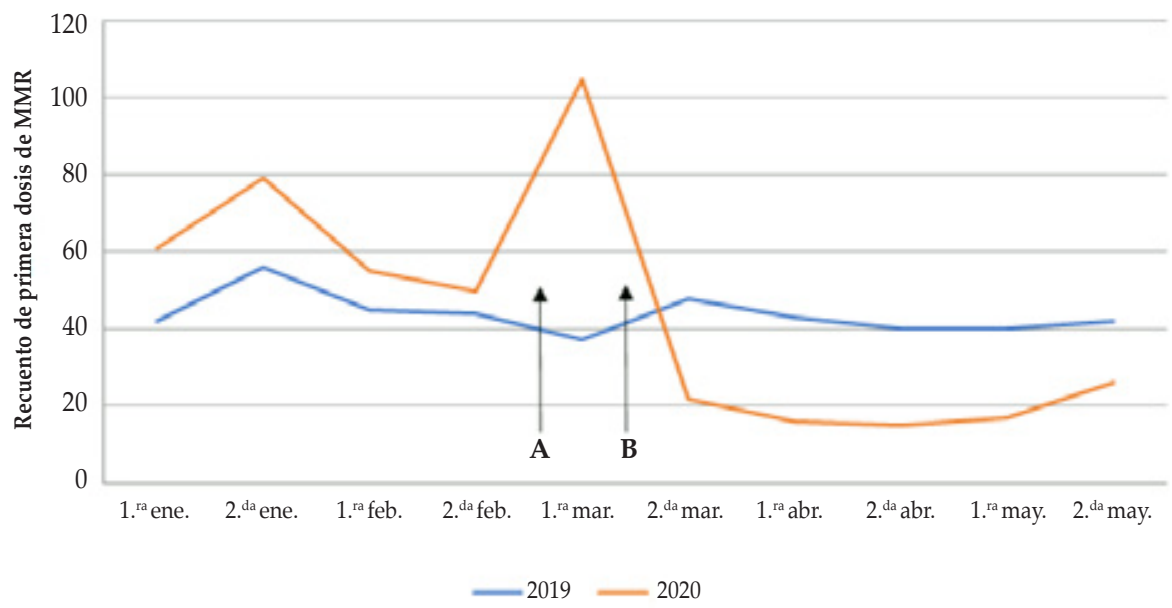

A. Comienzo de clases

B. Comienzo del aislamiento social preventivo. 
nuestro Hospital también disminuyó en el mismo período. ${ }^{8}$

Además, debe considerarse que la enfermedad pandémica generó temor en la población a concurrir a centros asistenciales. En una encuesta realizada por la Organización Mundial de la Salud-Fondo de las Naciones Unidas para la Infancia (OMS-UNICEF) en junio de este año sobre las causas de la falta de vacunación, la renuencia de los padres a asistir a los centros de vacunación por temor a la exposición al COVID-19 fue la más referida, mientras que un tercio de los encuestados indicó otros problemas, como el transporte público limitado y las políticas de distanciamiento físico. ${ }^{9}$ También debe considerarse que el aislamiento en nuestro país fue considerablemente más prolongado e intenso que en otros. ${ }^{10}$

Frente a estas barreras, hay que destacar que nuestro país declaró la vacunación como una actividad esencial durante el aislamiento social preventivo y obligatorio. ${ }^{1}$ En este sentido, en nuestro Hospital, si bien se realizaron modificaciones referidas al ingreso de los pacientes ("triage" y Unidad Febril de Urgencias), el vacunatorio no presentó ninguna modificación en su funcionamiento (días, horario, acceso).

Como potencial limitación se debe considerar que estos datos reflejan la situación particular de una institución. Sin embargo, nuestro Hospital es un centro pediátrico de referencia que, habitualmente, recibe 500000 consultas y aplica más de 50000 vacunas al año.

Aún no se determinó completamente el impacto que la pandemia y el aislamiento social y preventivo pueden tener en los niños. Sin embargo, los datos registrados en cuanto a la aplicación de vacunas son preocupantes. Contar con esta información es de vital importancia para la toma de conductas al respecto, con el objetivo de evitar el riesgo de brotes por enfermedades que son prevenibles mediante vacunas.

\section{CONCLUSIÓN}

Desde la instauración del aislamiento social preventivo y obligatorio, se observó una disminución del $64,2 \%$ en las vacunas aplicadas respecto del mismo período en el año anterior.

\section{REFERENCIAS}

1. Decreto 297/2020. Aislamiento social preventivo y obligatorio. Boletín Oficial de la República Argentina. Ciudad de Buenos Aires, 19 de marzo de 2020. [Acceso:28 de octubrede2020].Disponibleen:https: / / www.boletinoficial. gob.ar/detalleAviso/ primera/227042/ 20200320.

2. Isba R, Edge R, Jenner R, Broughton E, et al. Where have all the children gone? Decreases in paediatric emergency department attendances at the start of the COVID-19 pandemic of 2020. Arch Dis Child. 2020; 105(7):704.

3. Snapiri O, Rosenberg Danziger C, Krause I, Kravarusic D, et al. Delayed diagnosis of paediatric appendicitis during the COVID-19 pandemic. Acta Paediatr. 2020; 109(8):1672-6.

4. McDonald H, Tessier E, White J, Woodruff M, et al. Early impact of the coronavirus disease (COVID-19) pandemic and physical distancing measures on routine childhood vaccinations in England, January to April 2020. Euro Surveill. 2020; 25(19):2000848.

5. Santoli JM, Lindley MC, DeSilva MB, Kharbanda EO, et al. Effects of the COVID-19 Pandemic on Routine Pediatric Vaccine Ordering and Administration- United States, 2020. MMWR Morb Mortal Wkly Rep. 2020; 69(19):591-3.

6. Bramer CA, Kimmins LM, Swanson R, Kuo J, et al. Decline in Child Vaccination Coverage During the COVID-19 Pandemic - Michigan Care Improvement Registry, May 2016-May 2020. MMWR Morb Mortal Wkly Rep. 2020; 69(20):630-1.

7. Godoy K. Por qué eligen Casa Cuna los padres de los niños que no residen en su área de influencia. Rev Pediatr Elizalde. 2014; 5(2):57-132.

8. Ferrero F, Ossorio MF, Torres FA, Debaisi G. Impact of the COVID-19 pandemic in the paediatric emergency department attendances in Argentina. Arch Dis Child. 2020. [En prensa]

9. World Health Organization; UNICEF. IMMUNIZATION COVERAGE: ARE WE LOSING GROUND? Julio de 2020. [Acceso: 28 de octubre de 2020]. Disponible en: https: / / data.unicef.org/wp-content/uploads / 2020/07/ WUENIC-Immunization-coverage-are-we-losing-groundbrochure-2020.pdf.

10. Pieper O. Coronavirus: Argentina's never-ending quarantine. Deutsche Welle. (Bonn, DEU). 2020 Aug 27. [Acceso: 28 de octubre de 2020]. Disponible en: https:/ / www.dw.com/en/ coronavirus-argentinas-never-endingquarantine/a-54721129. 